\title{
EDITORIAL
}

\section{Importancia de los revisores de Revista Chilena de Radiología}

La labor de la Revista Chilena de Radiología de difundir la actividad académica generada en Chile y en países de Hispanoamérica ha sido apoyada desde sus inicios por la Sociedad Chilena de Radiología. Parte de los lineamientos desde su creación radica en ser el medio oficial de difusión de las actividades de la Sociedad en lo referido a producción científica. Hemos visto como ha ido creciendo la participación de las distintas instituciones en nuestro Congreso anual, y algunos de dichos trabajos también reciben difusión en nuestra Revista. Creemos que es una tarea ineludible fortalecer la producción de nuestros países, y permitir la extensión en las redes disponibles para que el conocimiento esté disponible para colegas radiólogos y médicos en período de formación. Dentro de estas redes tenemos la Revista Virtual del Colegio Interamericano de Radiología, que permite acceder sin costo a publicaciones de calidad de los afiliados al Colegio, y además la iniciativa Go-RAD, parte de la ISR (International Society of Radiology) donde artículos seleccionados de nuestra Revista, se hacen disponibles al mundo traducidos al inglés juntos a los artículos seleccionados de las mayores revistas de la especialidad.

Todo esto no sería posible sin la participación activa y desinteresada de los revisores que apoyan el accionar del Comité Editorial de la Revista Chilena de Radiología. Ellos, con su opinión experta entregada en forma ad-honorem, y sólo movidos por la pasión que implica el poder aportar al crecimiento de nuestra especialidad, permiten tomar la mejor decisión respecto de la calidad y utilidad de los trabajos presentados a consideración para publicación. Cada uno de ellos, revisa los manuscritos en forma "ciega" o blindada, de forma de poder opinar de forma absolutamente imparcial, referido a los méritos y las posibles correcciones que permitan una mejor entrega. Sin el aporte que ellos entregan en cada número, nuestra Revista no podría proporcionar un producto de calidad. Es por ello, que en este último número del año 2012, hacemos un reconocimiento y un agradecimiento por la labor y el tiempo que dedican a nuestra Revista.

Personalmente además quiero agradecer al Comité Editorial por todo el trabajo y dedicación entregado durante este año, y en particular al Dr. Andrés O'Brian quien en su rol de Editor Adjunto asumió la dirección de los dos últimos números del 2012, dada mi ausencia por una estadía en el extranjero. La labor silenciosa de todos permite que la Revista siga viva y creciendo, y esperamos incorporar a más y valiosos miembros durante el próximo año.

Gracias a todos y un feliz año 2013!

Dr. Claudio Silva F-A

Editor Científico 


\section{AGRADECIMIENTO A NUESTROS MÉDICOS REVISORES}

El trabajo de revisión de pares expertos es de la mayor importancia para proveer información actualizada de las distintas áreas de nuestra especialidad y así poder tomar decisiones adecuadas respecto de la inclusión de un artículo a publicación. Además, permite proporcionar a los autores retroalimentación valiosa de cómo poder mejorar un artículo, lo que es el reflejo de horas de arduo trabajo por parte de los autores. Esta labor es desinteresada y ad-honorem, sólo motivada por el interés de un mayor crecimiento de nuestra especialidad. Sus aportes son fundamentales para poder seguir creciendo en calidad y prestigio, y son la base sobre la cual se sostiene nuestra Revista. Es por ello, que Revista Chilena de Radiología quiere agradecer a todos aquellos que participaron en esta noble labor durante el año 2012.

Revisores

\begin{tabular}{|c|c|c|c|}
\hline Julia & Alegría B. & Andrés & O'Brien S. \\
\hline Claudia & Astudillo A. & Patricia & Orellana P. \\
\hline Álvaro & Belmar B. & Dulia & Ortega T. \\
\hline Patricia & Bitar $\mathrm{H}$. & Lizbeth & Pérez M. \\
\hline Álvaro & Burdiles $\mathrm{O}$. & Alejandra & Pizarro G. \\
\hline Francesca & Castoldi L. & Georgette & Pose L. \\
\hline Claudio & Cortés A. & Dravna & Razmilic V. \\
\hline Jorge & Díaz J. & Giancarlo & Schiappacasse F. \\
\hline Isabel & Fuentealba T. & Sergio & Soto $\mathrm{F}$. \\
\hline Gilberto & González V. & Ximena & Stecher G. \\
\hline Andrés & Labra W. & M. Loreto & Vergara del $\mathrm{R}$. \\
\hline David & Ladrón de Guevara & Alex & Wash F. \\
\hline Karla & Moënne B. & Nicolás & Zilleruelo V. \\
\hline Emilio & & & \\
\hline
\end{tabular}

